\title{
Sing Master: an Intelligent Mobile Game for Teaching Singing
}

\begin{abstract}
In this paper we present Sing Master - a new mobile application that allows singing enthusiasts to fulfil their dream: to learn singing. Sing Master is a game-like singing tutor, which provides continuous feedback on singers' pitch and timing. To this end, it presents a series of singing exercises build around musical intervals (e.g. major third). First, we motivate the use of software to evaluate and teach singing compared to a conventional singing coach. Next, we summarize some very similar software applications and outline what are the benefits of Sing Master compared to them. We finish by describing Sing Master's most useful feature to 'hear' the melody being sung and the algorithm, which 'hears' the musical pitch.
\end{abstract}

\section{INTRODUCTION}

Recent findings in psychology found that developing a music skill can be learned by anybody at any age [7]. Yet, it is timely and costly to find a singing teacher. For example, in small cities, there might be only very few people, capable of teaching singing. Furthermore, many users who want to learn singing, do not feel convinced that they have the potential to sing correctly. Thus they are not willing to make the step of taking a vocal training class with a singing teacher. Furthermore many users are simply shy to approach a singing teacher, because usually singing lessons are individual.

The demand to become a better singer is proven by the increasing number of downloaded apps that teach singing. For all these reasons a mobile smartphone application that teaches singing might be an appropriate time-saving and money-saving solution. Furthermore, singing is a desire: a singing enthusiast should not miss the moment of inspiration. The advantage of an application to a real-person singing coach is then that it is available anytime anywhere - for example allows practice at home.

\section{RELATED WORK}

Software applications for learning or enhancing singing skills are plenty. Very few of these applications however can 'hear' and interpret the actual sung voice. This allows a computer to provide visual feedback about some singing mistakes. Among the applications that achieve this are sing true and Choir Prodigy.

\section{A. Sing true}

Sing true [3] is a smart phone app that provides an intuitive way for practicing accurate pitch and volume. First off, it allows the user to improvise, singing freely. Then it allows him/her to learn based on analyzing the sung melody.

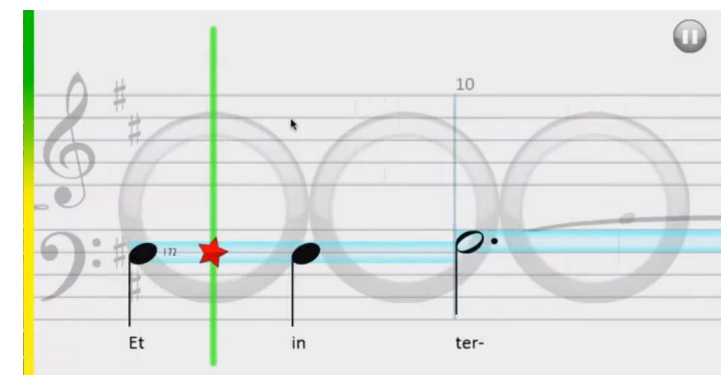

Fig. 1. Choir Prodigy visualizes scores for a given voice (for example bass). The current pitch of the voice is represented by a star

\section{B. Choir prodigy}

Choir prodigy [1] is a desktop software application that helps singers sing correctly a given piece. It provides the musical score for a selected voice from a complete musical score. It can understand the pitch of the main melody and visualize it real-time so that users can see if they are below or above the tone (fig. 1). A disadvatange of choir prodigy is though that no musical exercises are employed. Learning of important musical concepts like musical intervals is thus limited.

\section{DESCRIPTION}

In this work we present Sing Master: an open source smart phone application that allows a user to improve her/his singing skills in a systematic way. Description of the complete idea, updates and promotion video can be found at https://www. facebook.com/Singmasterapp. We have gathered a collection of practical exercises for training musical intervals adopted from [6]. Each exercise focuses on a musical interval - a major third, minor sixth etc.

\section{A. Exercise mode}

For a given exercise, reference tones are depicted by triangles on a 8-tone scale, which can be played by pressing the respective triangle. If an exercise is a major triad, for example, reference tones are the first third and fifth degree of the scale (fig.2). Because some beginner users might not be familiar with musical notation, musical notes are avoided. In other words, a musical scale is presented only by numbers in a vertical column. This is a clear and intuitive way for non-musicians.

To enhance the perception of the reference sounds, there is visual guidance as well: when the button REC to record is pressed, horizontal bars are colored simultaneously with the 


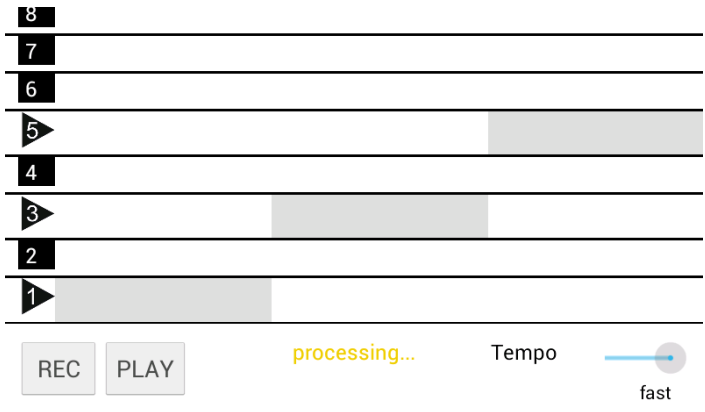

Fig. 2. Singing exercise on major triad. Reference tones: first, third and fifth degree of the scale are depicted by triangles on the western 8-tone major scale.

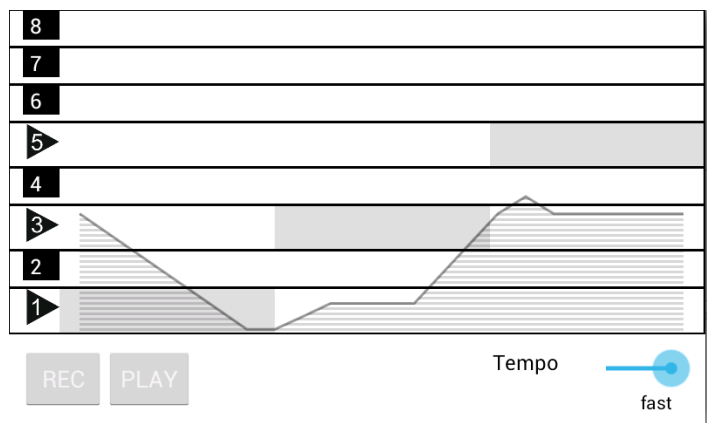

Fig. 3. Singing exercise on major triad. The detected pitch contour is visualized on top of the reference bars

tones to be sung. These bars are inspired by the well-known piano roll notation [5].

The most important feature of Sing Master is that it can 'hear' the melody of the singing voice: After recording, Sing Master gives visual feedback: the automatically extracted pitch of the voice is visualized as a dashed curve on top of the reference bars (fig. 3). Simultaneously, the recorded voice is played back. This way the user can see and hear where the mistakes are: where he/she sings off-tune and where the note onsets are sung with inaccurate timing.

As of now, a prototype of the exercise mode can be tested for Android on Google Play [2]. Further, to encourage reproducibility of this work and easy customization of the prototype, the source code is available under GPL license at https:/github.com/georgid/SINGmasterAndrioidWithGUI.

\section{B. Song mode}

Secondly, after each exercise Sing Master proposes a list of songs. Songs, for which a phrase from the melody has elements of the current exercise. Then you can select your favorite song and sing along the relevant section - for example only the chorus. This repeats while the level of difficulty increases.

Sing Master has as well a gaming element: it rates your performance - depending on how correct a melodic line is sung, Sing Master assigns a score from 1 to 100 . This way you can share your achievements in social networks and compare how well you sing your favorite songs with friends.
The Sing Master software is meant to be beneficial for everybody willing to learn singing: from absolute beginner to singing students in conservatories. By selecting more complex exercises and songs, Sing Master can be used as a tool to help professors in vocal training to assign homework exercises to their students.

\section{Pitch extraction}

To be able to 'hear' the melody of the singing voice, we apply Melodia - an algorithm that extracts main melody of singing voice in a robust way [8]. Melody extraction is the task of automatically estimating the fundamental frequency corresponding to the pitch of the predominant melodic line of a piece of polyphonic (or monophonic) music. In Melodia the extraction uses perceptual principles and assumptions. For example, one of these principles is that the pitch contour is continuous in time, i.e. does not include rapid changes or 'jumps'. We have used the implementation of Melodia as part of the open source framework for audio analysis Essentia [4].

\section{CONCLUSION}

In this work we have described a new mobile game application that can teach singing in a serendipitous, intuitive way. We have summarized as well some very similar software applications and outlined what are the benefits of Sing Master compared to them. We presented its unique feature to 'hear' the singing voice and evaluate the correctness of its pitch and timing. The advantage of the presented application is most of all its universality: it can be beneficial for everyone: from absolute beginners with no musical background to singing students in music conservatories.

Acknowledgements: We acknowledge financial support from the Spanish Ministry of Economy and Competitiveness, through the "María de Maeztu" Programme for Centres/Units of Excellence in R\&D” (MDM-2015-0502)

\section{REFERENCES}

[1] Choir prodigy. http://choirprodigy.com/. Accessed: 2015-10-18.

[2] Sing master. https://play.google.com/store/apps/details?id=bg.singmaster. gui\&hl=it. Accessed: 2015-10-18.

[3] singtrue. http://singtrue.co. Accessed: 2016-01-11.

[4] Dmitry Bogdanov, Nicolas Wack, E. Gómez, Sankalp Gulati, Perfecto Herrera, O. Mayor, Gerard Roma, Justin Salamon, J. R. Zapata, and $X$. Serra. Essentia: an audio analysis library for music information retrieval. In International Society for Music Information Retrieval Conference (ISMIR'13), pages 493-498, Curitiba, Brazil, 04/11/2013 2013.

[5] Donald Byrd. Music-notation searching and digital libraries. In Proceedings of the 1st ACM/IEEE-CS joint conference on Digital libraries, pages 239-246. ACM, 2001.

[6] Rosemary N Killam, Paul V Lorton, and Earl D Schubert. Interval recognition: Identification of harmonic and melodic intervals. Journal of Music Theory, pages 212-234, 1975.

[7] Gary F Marcus. Guitar zero: The new musician and the science of learning. Oneworld Publications, 2012.

[8] Justin Salamon and Emilia Gómez. Melody extraction from polyphonic music signals using pitch contour characteristics. Audio, Speech, and Language Processing, IEEE Transactions on, 20(6):1759-1770, 2012. 